\title{
Numerical simulation and experimental validation of heat transfer within rotating flows for three-dimensional non-axisymmetric, turbulent conditions
}

\author{
A. M. Raimundo*, , L. A. Oliveira and A. R. Figueiredo \\ Departamento Engenharia Mecânica, Universidade de Coimbra-Polo II, 3030-201 Coimbra, Portugal
}

\begin{abstract}
SUMMARY
A control volume type numerical methodology for the analysis of steady three-dimensional rotating flows with heat transfer, in both laminar and turbulent conditions, is implemented and experimentally tested. Non-axisymmetric momentum and heat transfer phenomena are allowed for. Turbulent transport is alternatively represented through three existing versions of the $k-\varepsilon$ model that were adjusted to take into account the turbulence anisotropy promoted by rotation, streamline curvature and thermal buoyancy. Their relative performance is evaluated by comparison of calculated local and global heat balances with those obtained through measurements in a laboratory device. A modified version of the Lam and Bremhorst, low Reynolds number model is seen to give the best results. A preliminary analysis focused on the flow structure and the transfer of heat is reported. Copyright (c) 2002 John Wiley \& Sons, Ltd.
\end{abstract}

KEY WORDS: rotating flow; non-axisymmetry; experimental results; CFD; heat transfer

\section{INTRODUCTION}

Non-isothermal rotating flows between parallel disks in a cylindrical geometry, with or without superposition of an axial throughflow, have intrinsic fundamental relevance. They are also encountered in many important practical applications: e.g. turbomachinery, lubrication, oceanography, computer hardware, gas turbines. In most of the available theoretical and experimental research works devoted to this subject axisymmetric conditions are assumed to prevail, e.g. References [1, 2]. However this simplifying hypothesis may not be realistic in many real working conditions. Namely, non-axisymmetric effects can be generated through association of thermal buoyancy with a non-vertical position of the axis of rotation or simply because the flow domain is defined by non-axisymmetric boundaries, e.g. the reading head of a computer disk. Few works are presently available where non-axisymmetry is allowed for, namely Long et al. [3] and Herrero et al. [4]. In all these studies the non-axisymmetry is

\footnotetext{
*Correspondence to: A. M. Raimundo, Departamento de Engenharia Mecânica, Polo Zda Universidade de Coimbra, Pinhal de Marrocos, 3030-201 Coimbra, Portugal.

†E-mail: antonio.raimundo@dem.uc.pt
} 


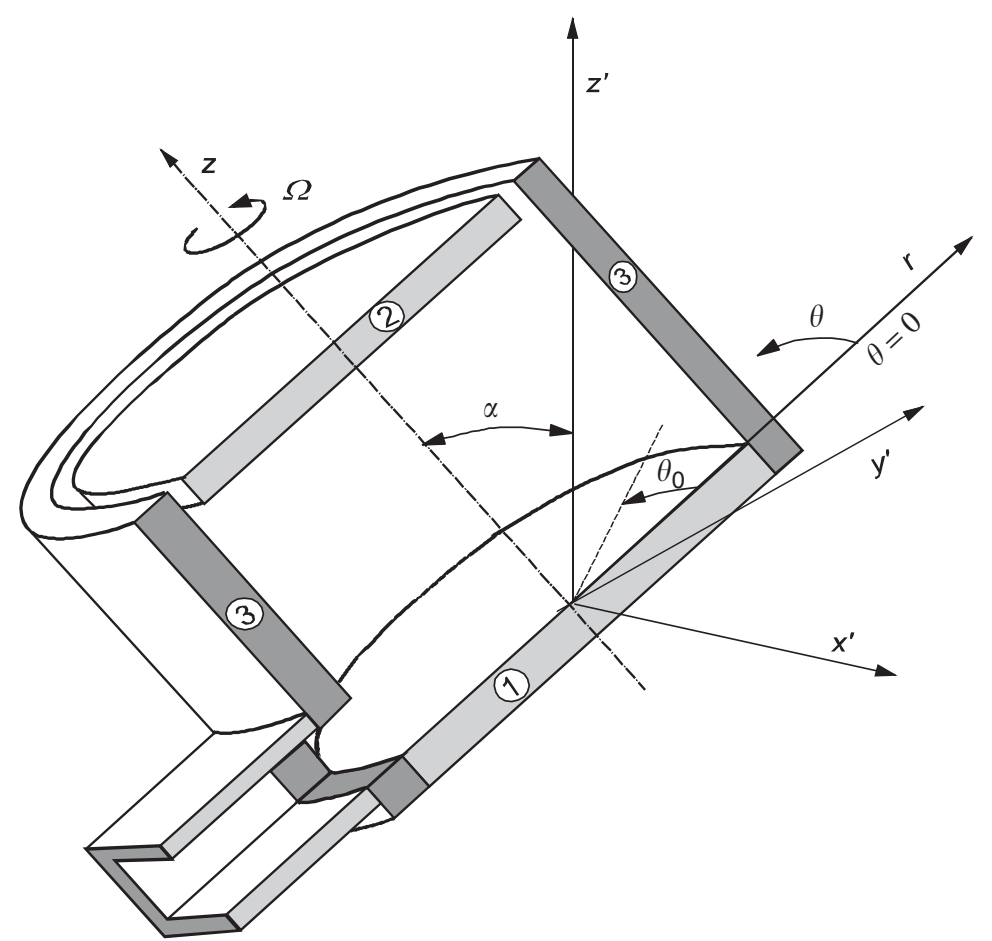

Figure 1. Schematic representation of the present geometry and co-ordinate system.

exclusively promoted by the thermal buoyancy forces. Besides, in those cases where a small axial flow is superimposed to the fluid rotation, re-ingress conditions may occur at the outlet section. This additional difficulty has been dealt with in few studies, e.g. References $[1,5,6]$. In most of these works the approach is experimental. Some of the authors have also used numerical models [5] and analytical methods based on the momentum-integral equations [6], but always with the restriction of axisymmetric conditions.

The use of a standard $k-\varepsilon$ approach to model the turbulent transport in rotating flows has a number of drawbacks that have been extensively reported in the literature [7]. On the other hand, the complexity and computational effort required by turbulent or algebraic stress modelling are not always worthy in terms of precision enhancement [8]. An interesting and simple alternative is the use of $k-\varepsilon$ low Reynolds models, on the condition that turbulence anisotropy promoted by rotation, streamline curvature and thermal buoyancy is adequately taken into consideration.

In the present work, the well-known finite-volume method, reinforced with some new calculation procedures, is used for the analysis of steady laminar or turbulent three-dimensional rotating flow of an incompressible (yet thermally dilatable) Newtonian fluid with heat transfer is presented and experimentally tested. A three-dimensional cylindrical co-ordinate system is used, thus allowing non-axisymmetric conditions to be considered. Numerical integration of the governing equations is performed through a control-volume type approach, where a staggered grid is used for the domain discretization. This requires the calculation of the radial 


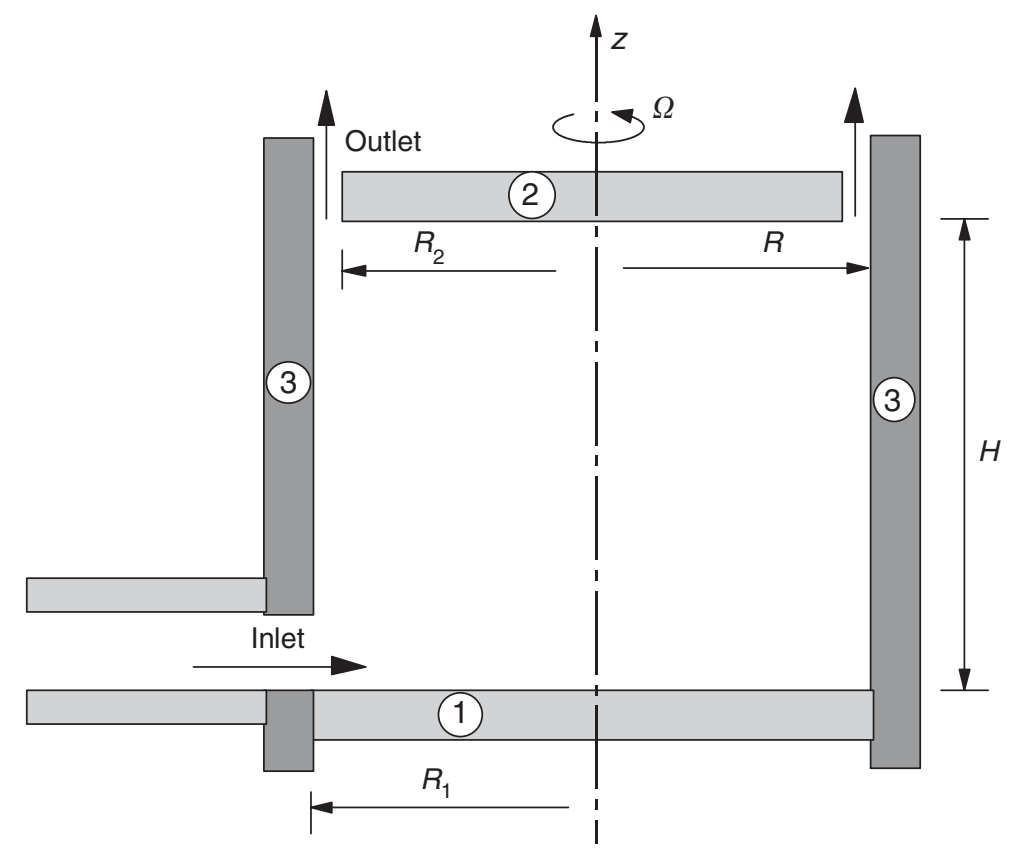

Figure 2. Relevant dimensions of the flow domain.

velocity component on the axis of rotation, the singularity in the corresponding differential equation being then numerically avoided through an original procedure. A specific numerical treatment is also developed to formulate the boundary conditions at outlet sections where fluid re-ingress occurs. Turbulent transport is represented by a $k-\varepsilon$ type approach, where three low-Reynolds number formulations [9-11] were slightly modified and alternatively used for comparison purposes. Evaluation of the theoretical method is performed through systematic comparisons of predicted local and global heat balances with the corresponding ones obtained in a parallel series of measurements that were conducted on a laboratory set-up, also described in this work, that allows the superposition of both rotation and throughflow effects. Satisfying agreement is provided by the modified Lam and Bremhorst model, which is thus used to establish a number of preliminary considerations upon the flow structure and the transport of heat. A systematic analysis of these features will be published in the near future.

The basic configuration of the flow domain is shown schematically in Figures 1 and 2. The working fluid (air) is enclosed by two parallel coaxial disks (1 and 2) and a cylindrical shroud (3). The cavity may be oriented in any space direction. The fluid is admitted into the domain through a radially oriented opening of rectangular cross-section and leaves it axially through a ring like clearance between the disk 2 and the cylindrical shroud.

\section{THEORETICAL MODEL}

The governing transport equations of steady three-dimensional turbulent flow are applied in their $(r, \theta, z)$ cylindrical co-ordinate form, where $z$ is the axis of rotation (see Figure 1). 
Table I. Values of $\Gamma_{\phi}$ and $S_{\phi}$ in the general transport equation.

\begin{tabular}{lccc}
\hline Transport property & $\phi$ & $\Gamma_{\phi}$ & $S_{\phi}$ \\
\hline Mass & 1 & 0 & 0 \\
Momentum in $r$ direction & $U$ & $\mu_{\mathrm{ef}}=\mu+\mu_{\mathrm{t}}$ & $-\frac{\partial P}{\partial r}+S_{U}+B_{U}$ \\
Momentum in $\theta$ direction & $V$ & $\mu_{\mathrm{ef}}=\mu+\mu_{\mathrm{t}}$ & $-\frac{1}{r} \frac{\partial P}{\partial \theta}+S_{V}+B_{V}$ \\
Momentum in $z$ direction & $W$ & $\mu_{\mathrm{ef}}=\mu+\mu_{\mathrm{t}}$ & $-\frac{\partial P}{\partial z}+S_{W}+B_{W}$ \\
Thermal energy & $T$ & $\Gamma^{T}=\frac{\mu}{P r}+\frac{\mu_{\mathrm{t}}}{P r_{\mathrm{t}}}$ & $S_{T}$ \\
Turbulent kinetic energy & $k$ & $\Gamma^{k}=\mu+\frac{\mu_{\mathrm{t}}}{\sigma_{k}}$ & $P_{k}+G_{k}-D_{k}-B_{k}$ \\
Dissipation rate of $k\left(\tilde{\varepsilon}=\varepsilon-B_{k}\right)$ & $\tilde{\varepsilon}$ & $\Gamma^{\varepsilon}=\mu+\frac{\mu_{\mathrm{t}}}{\sigma_{\varepsilon}}$ & $P_{\varepsilon}+G_{\varepsilon}-D_{\varepsilon}+E_{\varepsilon}+Y_{\varepsilon}$ \\
\hline
\end{tabular}

To describe the arbitrary orientation of the cavity, an auxiliary Cartesian co-ordinate system $\left(x^{\prime}, y^{\prime}, z^{\prime}\right)$, is also used, where the angle between the vertical axis $z^{\prime}$ and the axis of rotation is noted $\alpha$. All time-averaged equations representing conservation of a general-dependent variable $\phi$ may be cast into the following common form:

$$
\begin{aligned}
\frac{1}{r} & \frac{\partial}{\partial r}(r \rho U \phi)+\frac{1}{r} \frac{\partial}{\partial \theta}(\rho V \phi)+\frac{\partial}{\partial z}(\rho W \phi) \\
\quad & \frac{1}{r} \frac{\partial}{\partial r}\left(r \Gamma_{\phi} \frac{\partial \phi}{\partial r}\right)+\frac{1}{r^{2}} \frac{\partial}{\partial \theta}\left(\Gamma_{\phi} \frac{\partial \phi}{\partial \theta}\right)+\frac{\partial}{\partial z}\left(\Gamma_{\phi} \frac{\partial \phi}{\partial z}\right)+S_{\phi}
\end{aligned}
$$

where $U, V, W$ are the mean flow velocity components along the $r, \theta$ and $z$ directions, respectively. The diffusivity $\Gamma_{\phi}$ and the source $S_{\phi}$ have different expressions for each variable $\phi$ as indicated in Table I.

The possible occurrence of significant temperature gradients within the flow renders the Boussinesq hypothesis inadequate for the present purposes [12]. Thus the fluid density $\rho$, dynamic viscosity $\mu$, thermal conductivity $\lambda$, heat capacity $c_{\mathrm{p}}$ and thermal expansion coefficient $\beta$ are all taken at the local fluid temperature $T$, according to the relations given by Zografos et al. [13]. In Table I, $P=P_{\mathrm{r}}+\left(\frac{2}{3}\right) \rho k$ stands for an effective pressure, where $P_{\mathrm{r}}$ is the reduced pressure $[14,15]$. The momentum $S_{U}, S_{V}, S_{W}$, and thermal buoyancy $B_{U}, B_{V}$, $B_{W}$ source contributions (see also Figure 1) are defined in the following expressions, where $g=+9.81 \mathrm{~m} / \mathrm{s}^{2}$

$$
S_{U}=\frac{1}{r} \frac{\partial}{\partial r}\left(r \mu_{\mathrm{ef}} \frac{\partial U}{\partial r}\right)+\frac{1}{r} \frac{\partial}{\partial \theta}\left(\mu_{\mathrm{ef}} \frac{\partial V}{\partial r}\right)+\frac{\partial}{\partial z}\left(\mu_{\mathrm{ef}} \frac{\partial W}{\partial r}\right)
$$


HEAT TRANSFER IN 3D NON-AXISYMMETRIC ROTATING FLOWS

Table II. Empirical constants for the low Reynolds $k-\varepsilon$ models.

\begin{tabular}{lccccccc}
\hline Model & $C_{\mu}$ & $C_{1}$ & $C_{2}$ & $C_{3}$ & $\sigma_{k}$ & $\sigma_{\varepsilon}$ & $P r_{\mathrm{t}}$ \\
\hline LS & 0.09 & 1.44 & 1.92 & 1.44 & 1.0 & 1.3 & 0.9 \\
LB & 0.09 & 1.44 & 1.92 & 1.44 & 1.0 & 1.3 & 0.9 \\
LD & 0.09 & 1.44 & 1.92 & 1.44 & 1.0 & 1.3 & 0.9 \\
\hline
\end{tabular}

$$
\begin{gathered}
+\rho \frac{V^{2}}{r}-2 \mu_{\mathrm{ef}} \frac{U}{r^{2}}-3 \frac{\mu_{\mathrm{ef}}}{r^{2}} \frac{\partial V}{\partial \theta}-\frac{V}{r^{2}} \frac{\partial \mu_{\mathrm{ef}}}{\partial \theta} \\
S_{V}=\frac{\partial}{\partial r}\left(\frac{\mu_{\mathrm{ef}}}{r} \frac{\partial U}{\partial \theta}\right)+\frac{1}{r} \frac{\partial}{\partial \theta}\left(\frac{\mu_{\mathrm{ef}}}{r} \frac{\partial V}{\partial \theta}\right)+\frac{\partial}{\partial z}\left(\frac{\mu_{\mathrm{ef}}}{r} \frac{\partial W}{\partial \theta}\right) \\
-\rho \frac{U V}{r}-\mu_{\mathrm{ef}} \frac{V}{r^{2}}+4 \frac{\mu_{\mathrm{ef}}}{r^{2}} \frac{\partial U}{\partial \theta}-\frac{V}{r} \frac{\partial \mu_{\mathrm{ef}}}{\partial r}+2 \frac{U}{r^{2}} \frac{\partial \mu_{\mathrm{ef}}}{\partial \theta} \\
S_{W}=\frac{1}{r} \frac{\partial}{\partial r}\left(r \mu_{\mathrm{ef}} \frac{\partial U}{\partial z}\right)+\frac{1}{r} \frac{\partial}{\partial \theta}\left(\mu_{\mathrm{ef}} \frac{\partial V}{\partial z}\right)+\frac{\partial}{\partial z}\left(\mu_{\mathrm{ef}} \frac{\partial W}{\partial z}\right) \\
B_{U}=-\left(\rho-\rho_{0}\right) g \sin \alpha \cos \left(\theta-\theta_{0}\right) \\
B_{V}=\left(\rho-\rho_{0}\right) g \sin \alpha \sin \left(\theta-\theta_{0}\right) \\
B_{W}=-\left(\rho-\rho_{0}\right) g \cos \alpha
\end{gathered}
$$

where $\theta_{0}$ is the angular position of the intersection of the vertical plane containing the axes $z$ and $z^{\prime}$ with the inside surface of disk 1 .

The dissipation source $S_{T}$ is due to viscosity and turbulence effects. In its expression

$$
S_{T}=\frac{1}{c_{\mathrm{p}}}(\mu \Phi+\rho \varepsilon)
$$

$\varepsilon$ is the rate of dissipation of the turbulent kinetic energy $k$ and $\Phi$ is the dissipation function.

For computational reasons, it is convenient to use a newly defined dissipation variable $\tilde{\varepsilon}=\varepsilon-B_{k}$, where $B_{k}$ is the value taken by $\varepsilon$ at a solid wall. This allows one to set $\tilde{\varepsilon}=0$ as the corresponding boundary condition. Thus the Prandtl-Kolmogorov relation for turbulent viscosity becomes

$$
\mu_{\mathrm{t}}=C_{\mu} f_{\mu} \rho \frac{k^{2}}{\tilde{\varepsilon}}
$$

The empirical constants and the damping functions to be used in the different turbulence models [Launder and Sharma (LS), Lam and Bremhorst (LB) and Lars Davidson (LD)] are specified in Tables II and III, respectively. Two-dimensionless parameters are necessary to 
Table III. Damping functions for the low Reynolds $k-\varepsilon$ models.

\begin{tabular}{lccc}
\hline Model & $f_{\mu}$ & $f_{1}$ & $f_{2}$ \\
\hline LS & $\exp \left[\frac{-3.4}{\left(1+R e_{\mathrm{t}} / 50\right)^{2}}\right]$ & 1.0 & $1-0.3 \exp \left(-R e_{\mathrm{t}}^{2}\right)$ \\
LB & {$\left[1-\exp \left(-0.0165 R e_{k}\right)\right]^{2}\left(1+\frac{20.5}{R e_{\mathrm{t}}}\right)$} & $1+\left(\frac{0.05}{f_{\mu}}\right)^{3}$ & $1-\exp \left(-R e_{\mathrm{t}}^{2}\right)$ \\
LD & $\exp \left[\frac{-3.4}{\left(1+R e_{\mathrm{t}} / 50\right)^{2}}\right]$ & $1+\left(\frac{0.14}{f_{\mu}}\right)^{3}$ & {$\left[1-0.27 \exp \left(-R e_{\mathrm{t}}^{2}\right)\right]$} \\
\hline
\end{tabular}

define the damping functions, namely the turbulence Reynolds number $R e_{\mathrm{t}}$ and the near wall, local turbulent Reynolds number $\operatorname{Re}_{k}$ :

$$
R e_{\mathrm{t}}=\frac{\rho k^{2}}{\mu \tilde{\varepsilon}} \quad \text { and } \quad R e_{k}=\frac{\rho y_{\mathrm{n}} k^{1 / 2}}{\mu}
$$

where $y_{\mathrm{n}}$ is the normal distance to the wall.

In Table I, the term of energy production due to turbulent stresses $P_{k}$, the dissipation term $D_{k}$ and the energy source contribution due to buoyancy $G_{k}$ are modelled through the use of the turbulent viscosity $\mu_{\mathrm{t}}$ as follows:

$$
\begin{aligned}
& P_{k}=\mu_{\mathrm{t}} \Phi \\
& D_{k}=\rho \tilde{\varepsilon}=C_{\mu} f_{\mu} \rho^{2} \frac{k^{2}}{\mu_{\mathrm{t}}}
\end{aligned}
$$

and

$$
G_{k}=\beta g \frac{\mu_{\mathrm{t}}}{P r_{\mathrm{t}}}\left[-\frac{\partial T}{\partial r} \sin \alpha \cos \left(\theta-\theta_{0}\right)+\frac{1}{r} \frac{\partial T}{\partial \theta} \sin \alpha \sin \left(\theta-\theta_{0}\right)-\frac{\partial T}{\partial z} \cos \alpha\right]
$$

Turbulence isotropy is admitted to hold in the basic versions of the three models used in this work. However, significant anisotropy may arise from rotation, streamline curvature and buoyancy effects and thus should not be ignored in the present conditions. The starting versions were than accordingly modified, as reported below.

Stable thermal stratification damps vertical and amplifies horizontal velocity fluctuations, while the inverse effect is promoted by unstable stratification. This was approximately represented through the introduction of a new function $f_{3}$ in the $G_{\varepsilon}$ buoyancy term for the $\tilde{\varepsilon}$ equation, to make it dependent on the local flow velocity direction, as suggested in Reference [16]:

$$
\begin{aligned}
G_{\varepsilon} & =C_{3} f_{3} \frac{\tilde{\varepsilon}}{k} G_{k} \\
f_{3} & =\tanh \left(\left|V_{\mathrm{V}}\right| /\left|V_{\mathrm{H}}\right|\right)
\end{aligned}
$$


According to the free orientation of the co-ordinate system represented in Figure 1, the absolute values of the vertical and horizontal components of velocity in Equation (11) have the following expressions:

$$
\begin{aligned}
& \left|V_{\mathrm{V}}\right|=\left|U \sin \alpha \cos \left(\theta-\theta_{0}\right)-V \sin \alpha \sin \left(\theta-\theta_{0}\right)+W \cos \alpha\right| \\
& \left|V_{\mathrm{H}}\right|=\sqrt{U^{2}+V^{2}+W^{2}-\left|V_{\mathrm{V}}\right|^{2}}
\end{aligned}
$$

As was pointed out by Launder et al. [17], streamline curvature tends to damp turbulence whenever the angular momentum increases with radius and to amplify it in the opposite case. Following those authors, these effects are here accounted for through the introduction of an empirical function $f_{\mathrm{c}}$ that is used to modify the dissipation term of the $\tilde{\varepsilon}$ equation:

$$
\begin{aligned}
D_{\varepsilon} & =C_{2} f_{2} f_{\mathrm{c}} \rho \frac{\tilde{\varepsilon}^{2}}{k} \\
f_{\mathrm{c}} & =1-C_{\mathrm{c}} \frac{k^{2}}{\tilde{\varepsilon}^{2}} \frac{V}{r^{2}} \frac{\partial}{\partial r}(r V)
\end{aligned}
$$

where the new constant $C_{\mathrm{c}}$ takes the value 0.2 , unless there is no external peripheral wall [(3) in Figure 1], for which case $C_{\mathrm{c}}=0.0$.

As suggested by Nallasamy [8], rotation has a stabilizing effect as it damps the energy transfer between large- and small-scale eddies. This is represented through the inclusion of a new empirical function $f_{\mathrm{r}}$ in the source production term of the $\tilde{\varepsilon}$ equation:

$$
\begin{aligned}
& P_{\varepsilon}=C_{1} f_{1} f_{\mathrm{r}} \frac{\tilde{\varepsilon}}{k} P_{k} \\
& f_{\mathrm{r}}=1+2 \frac{C_{\mathrm{r}}}{\Phi}\left(\frac{V}{r} \frac{\partial V}{\partial r}-\frac{V^{2}}{r^{2}}\right)
\end{aligned}
$$

The new constant $C_{\mathrm{r}}$ is equal to 0.9 whenever there is rotation and zero otherwise.

In order to compensate for the overestimation of the near wall turbulent length scales (a drawback common to all $k-\varepsilon$ type models where the $\varepsilon$ equation is actually solved up to the wall), Yap [18] suggested the introduction of a new source term $Y_{\varepsilon}$ in the $\tilde{\varepsilon}$ equation that implicitly limits the turbulent length scales:

$$
Y_{\varepsilon}=\max \left[C_{\mathrm{y}}\left(\frac{k^{3 / 2}}{\tilde{\varepsilon} C_{\ell} y_{\mathrm{n}}}-1.0\right)\left(\frac{k}{C_{\ell} y_{\mathrm{n}}}\right)^{2}, 0.0\right]
$$

The values adopted for the constants in this Yap correction were those leading to the best agreement with available laboratory measurements [15]: $C_{\ell}=2.548$ and $C_{\mathrm{y}}=0.83$.

The additional terms $B_{k}$ and $E_{\varepsilon}$ of Table I depend on the model to be considered [9-11] and are defined in Table IV, through the following auxiliary terms $B_{k}^{*}$ and $E_{\varepsilon}^{*}$ :

$$
B_{k}^{*}=\left(\frac{\partial k^{1 / 2}}{\partial r}\right)^{2}+\left(\frac{1}{r} \frac{\partial k^{1 / 2}}{\partial \theta}\right)^{2}+\left(\frac{\partial k^{1 / 2}}{\partial z}\right)^{2}
$$


Table IV. Extra terms and boundary conditions for the $k$ and $\tilde{\varepsilon}$ equations.

\begin{tabular}{lccccc}
\hline Model & Code & $B_{k}$ & $E_{\varepsilon}$ & $\begin{array}{c}\text { Boundary } \\
\text { conditions }(k)\end{array}$ & $\begin{array}{c}\text { Boundary } \\
\text { conditions }(\tilde{\varepsilon})\end{array}$ \\
\hline Launder and Sharma & LS & $2 \mu B_{k}^{*}$ & $2 \frac{\mu \mu_{\mathrm{t}}}{\rho} E_{\varepsilon}^{*}$ & $k_{\mathrm{w}}=0$ & $\tilde{\varepsilon}_{\mathrm{w}}=0$ \\
Lam and Bremhorst & LB & 0 & 0 & $k_{\mathrm{w}}=\partial k / \partial y_{\mathrm{n}}=0$ & $\partial \tilde{\varepsilon} / \partial y_{\mathrm{n}}=0$ \\
Lars Davidson & LD & 0 & 0 & $k_{\mathrm{w}}=0$ & $\partial \tilde{\varepsilon} / \partial y_{\mathrm{n}}=0$ \\
\hline
\end{tabular}

$$
\begin{aligned}
E_{\varepsilon}^{*}= & \left(\frac{\partial^{2} U}{\partial r^{2}}\right)^{2}+\left(\frac{1}{r^{2}} \frac{\partial^{2} U}{\partial \theta^{2}}+\frac{1}{r} \frac{\partial U}{\partial r}-\frac{U}{r^{2}}-\frac{2}{r^{2}} \frac{\partial V}{\partial \theta}\right)^{2}+\left(\frac{\partial^{2} U}{\partial z^{2}}\right)^{2} \\
& +\left(\frac{\partial^{2} V}{\partial r^{2}}\right)^{2}+\left(\frac{1}{r^{2}} \frac{\partial^{2} V}{\partial \theta^{2}}+\frac{1}{r} \frac{\partial V}{\partial r}-\frac{V}{r^{2}}+\frac{2}{r^{2}} \frac{\partial U}{\partial \theta}\right)^{2}+\left(\frac{\partial^{2} V}{\partial z^{2}}\right)^{2} \\
& +\left(\frac{\partial^{2} W}{\partial r^{2}}\right)^{2}+\left(\frac{1}{r^{2}} \frac{\partial^{2} W}{\partial \theta^{2}}+\frac{1}{r} \frac{\partial W}{\partial r}\right)^{2}+\left(\frac{\partial^{2} W}{\partial z^{2}}\right)^{2}+2 E^{*}
\end{aligned}
$$

The term $E^{*}$ in this latter equation involves crossed space, second-order derivatives and is thus discarded.

At solid walls, velocity and temperature boundary conditions are set by locally imposing Dirichlet and Neumann type conditions, respectively. The boundary conditions for $k$ and $\tilde{\varepsilon}$ are showed in Table IV for the low Reynolds number models considered in this work.

For free boundaries recirculation may occur and a special numerical treatment is thus necessary; besides, Equation (1) leads to a mathematical singularity at the axis of rotation $(r=0)$, that may be overcome through a particular numerical strategy. Both procedures will be presented in the next section. For those cases where axial symmetry holds, conditions at $r=0$ are simply reduced to $U=0$ with zero radial gradient for the other variables.

Flow inlet conditions are those corresponding to fully developed flow in the admission duct. When this flow is turbulent, the fluctuating level is locally set by defining a turbulence inlet intensity $I_{\mathrm{t}, \text { in }}$, thus leading to

$$
k_{\text {in }}=1.5 I_{\mathrm{t}, \text { in }}^{2} V_{\text {in }}^{2}
$$

and

$$
\varepsilon_{\text {in }}=\frac{C_{\mu}^{0.75}}{\ell_{\mathrm{m}}} k_{\mathrm{in}}^{1.5}
$$

where $V_{\text {in }}$ is the inlet mean velocity and $\ell_{\mathrm{m}}$ is the turbulent mixing length. For fully developed jets like this one, Costa et al. [16] have measured local turbulence intensities between 0.02 and 0.1. Exploratory calculations [15], indicated that, for levels of $I_{\mathrm{t}, \text { in }}<0.1$, the numerical solutions were nearly insensitive to the assumed distributions of $k$ and $\varepsilon$ at the inlet section. A value of $I_{\mathrm{t}, \text { in }}=0.04$ is thus assumed for the present conditions. The fluid temperature $T_{\text {in }}$ in is considered uniform in the whole inlet section.

Outflow boundary conditions are not known a priori and may involve flow recirculation in some cases. This is dealt with by use of a special numerical procedure, as reported in the next section. 


\section{NUMERICAL METHOD}

Numerical integration of Equation (1) for the conservation of mass $(\phi=1)$, momentum $(\phi=U, V, W)$, thermal energy $(\phi=T)$, turbulent kinetic energy $(\phi=k)$ and its (modified) rate of dissipation $(\phi=\tilde{\varepsilon})$ is performed through a control volume based, finite difference approach, where hybrid differencing is used for the diffusive and advective terms. The wellestablished general procedure was extensively described, e.g. by Patankar [19]. This text is thus exclusively focused on the particular strategies that were conceived and/or implemented for the specific present purposes.

Discretization of the flow domain has to ensure that grid refinement is sufficient to cope with those regions corresponding to the highest flow gradients. Besides, for low Reynolds turbulence models the grid nodes immediately adjacent to each wall must be located well inside its viscous sublayer (thus meaning a dimensionless distance from the wall $y^{+} \ll 11$ ). The use of a hyperbolic tangent function is particularly efficient in defining an irregularly spaced mesh that satisfies those fundamental requirements with a relatively small number of total grid nodes, thus keeping the necessary CPU time and memory within reasonable limits.

For grid independence analysis, a test-case with rotating and mass flow rate Reynolds numbers of $R e_{\Omega}=144.0 \times 10^{3}$ and $R e_{\mathrm{m}}=284.32$, respectively, was chosen (both these parameters are defined in Section 5). Numerical tests with non-uniform grids ranging between $12 \times 20 \times 12$ and $30 \times 46 \times 30$ nodes, along the $r, \theta$ and $z$ directions, respectively, showed that a grid of $20 \times 30 \times 20$ nodes yielded a nearly grid-independent solution for this test-case. Thus, an irregular distribution of $20 \times 30 \times 20$ nodes was adopted for the domain discretization.

Scalar and back staggered control volumes are specified along the $r, \theta$ and $z$ directions through the indices $I, J, K$, respectively. The flow domain includes all scalar nodes with $(2 \leqslant I \leqslant N I-1),(2 \leqslant J \leqslant N J-1),(2 \leqslant K \leqslant N K-1)$. The scalar fictitious nodes $1, N I, N J, N K$ are placed outside the flow domain. For a typical control volume surrounding a node $\mathrm{P}$, the algebraic equivalent of Equation (1) is

$$
a_{\mathrm{P}}^{\phi} \phi_{\mathrm{P}}=a_{\mathrm{N}}^{\phi} \phi_{\mathrm{N}}+a_{\mathrm{S}}^{\phi} \phi_{\mathrm{S}}+a_{\mathrm{E}}^{\phi} \phi_{\mathrm{E}}+a_{\mathrm{W}}^{\phi} \phi_{\mathrm{W}}+a_{\mathrm{T}}^{\phi} \phi_{\mathrm{T}}+a_{\mathrm{B}}^{\phi} \phi_{\mathrm{B}}+b_{\mathrm{P}}^{\phi}
$$

where $a^{\phi}$ are weighting factors, the indices N, S, E, W, T, B stand for North, South, East, West, Top and Bottom, respectively, and $b_{\mathrm{P}}^{\phi}$ is an independent additional term resulting from linearization of the source term $S_{\phi}$ in Equation (1). Temperature, pressure, turbulent kinetic energy and its rate of dissipation are calculated at the main grid nodes while staggered grids (located at the main control volume faces) are used for the three velocity components. The SIMPLEC segregated algorithm of van Doormaal and Raithby [20] is adopted for the whole numerical procedure, the solution for the individual $\phi$ equation sets being obtained through a line-by-line iteration procedure based on the well known three-diagonal matrix algorithm (TDMA). Convergence is satisfied when the sum of all nodal normalized residuals is less than $10^{-6}$ for the mass balance and less than $10^{-5}$ for the remaining equations. For an irregularly spaced mesh of $(20 \times 30 \times 20)$ nodes along the $r, \theta$ and $z$ directions, respectively, this is typically achieved after 14000 iterations, corresponding to about $4 \mathrm{~h} \mathrm{CPU}$ time on a standard PC with a $450 \mathrm{MHz}$ Pentium II processor.

Even though most of the adopted methodologies are now well established, a number of special procedures proved to be crucial for the present needs. A summary of those procedures, for which additional details may be found in Raimundo [15], is now presented. 
Outlet flow boundaries - When the superimposed throughflow is not large enough, fluid re-ingress may occur at the outlet boundary (see Figure 2), thus rendering zero gradient type boundary conditions inappropriate. This is overcome by locally imposing mass and thermal energy conservation in each control volume of the boundary. As an example, the axial velocity component at the fictitious node $N K$ outside the East boundary is determined through a mass balance applied to the corresponding scalar control volume localized at node $N K-1$, where $N K$ is the number of grid nodes in the axial $(z)$ direction. Once this procedure is completed for all outlet boundary nodes, a final multiplying correction is applied to ensure global mass and energy conservation.

Mathematical singularity at $r=0$ - As a consequence of the back staggered grid adopted in the present formulation, the radial velocity component $U$ of the first real node in the radial direction $(I=2)$ is localized at $r=0$, thus leading to a singularity in the corresponding Equation (1). This problem is simply overcome by displacing the node $I=2$ of the staggered $U$ grid from $r=0$ to a position midway between the $z$-axis and the node surface $I=2$ of the scalar grid (local semi-staggered grid).

Line-by-line iteration procedure for the $\theta$ direction ('Circular' TDMA)-Resolution of Equation (23) in the $\theta$ direction cannot be adequately performed through the use of a conventional TDMA procedure. In fact, for fixed $I$ and $K$, the fictitious nodes 1 and $N J$ coincide with the real nodes $N J-1$ and 2, respectively. This difficulty was overcome through the introduction of a modified algorithm, called 'Circular' TDMA (CTDMA), that is fully described in Raimundo [15].

Reliable numerical and/or experimental data to be used as a reference for comparison with the present numerical predictions is still lacking in the available literature. Validation of this theoretical model was thus performed for a number of test cases through comparison of calculated results with the corresponding measurements obtained in the experimental rig that is described in the next section.

\section{EXPERIMENTAL APPARATUS}

The experimental set-up is essentially made of the components schematically shown in Figure 2, with $\alpha=0$ : a cylindrical cavity with a horizontal aluminium hot disk (1) at the bottom, a Plexiglas rotating disk (2) at the top, a fixed cylindrical Plexiglas shroud (3) and a horizontal duct of rectangular cross-section introducing a radially directed jet of air into the cavity. As sketched in Figure 2, the flow domain dimensions are $H=202 \mathrm{~mm}$, $R=R_{1}=204 \mathrm{~mm}, R_{2}=200 \mathrm{~mm}$ and the rectangular duct has an internal cross-section of $50 \mathrm{~mm}$ height and $20 \mathrm{~mm}$ width. Additional details may be found in Reference [15].

The fixed disk is heated by means of a sandwich-type construction, schematically presented in Figure 3(b). The sandwich is an assembly of five disks with similar dimensions: two aluminium disks at the top (1) and bottom (7) extremes and three insulation bakelite disks (4)-(6) in between. Disk (1) is equipped with thermocouples and heated by means of an independent, calibrated electrical resistance (9) of $R_{9}=208.83 \Omega$. Disk (7) has a role of thermal compensation. It is heated by an electrical resistance (10) of $R_{10}=200.41 \Omega$. During the construction of disk (1), a circular sector was selected and cut off. This sector was then equipped with an independent resistance (8) of $R_{8}=2.70 \Omega$ together with several thermocouples and one $0.5 \mathrm{~mm}$ thick insulating plastic trip on each side. Finally, it was reinserted at 

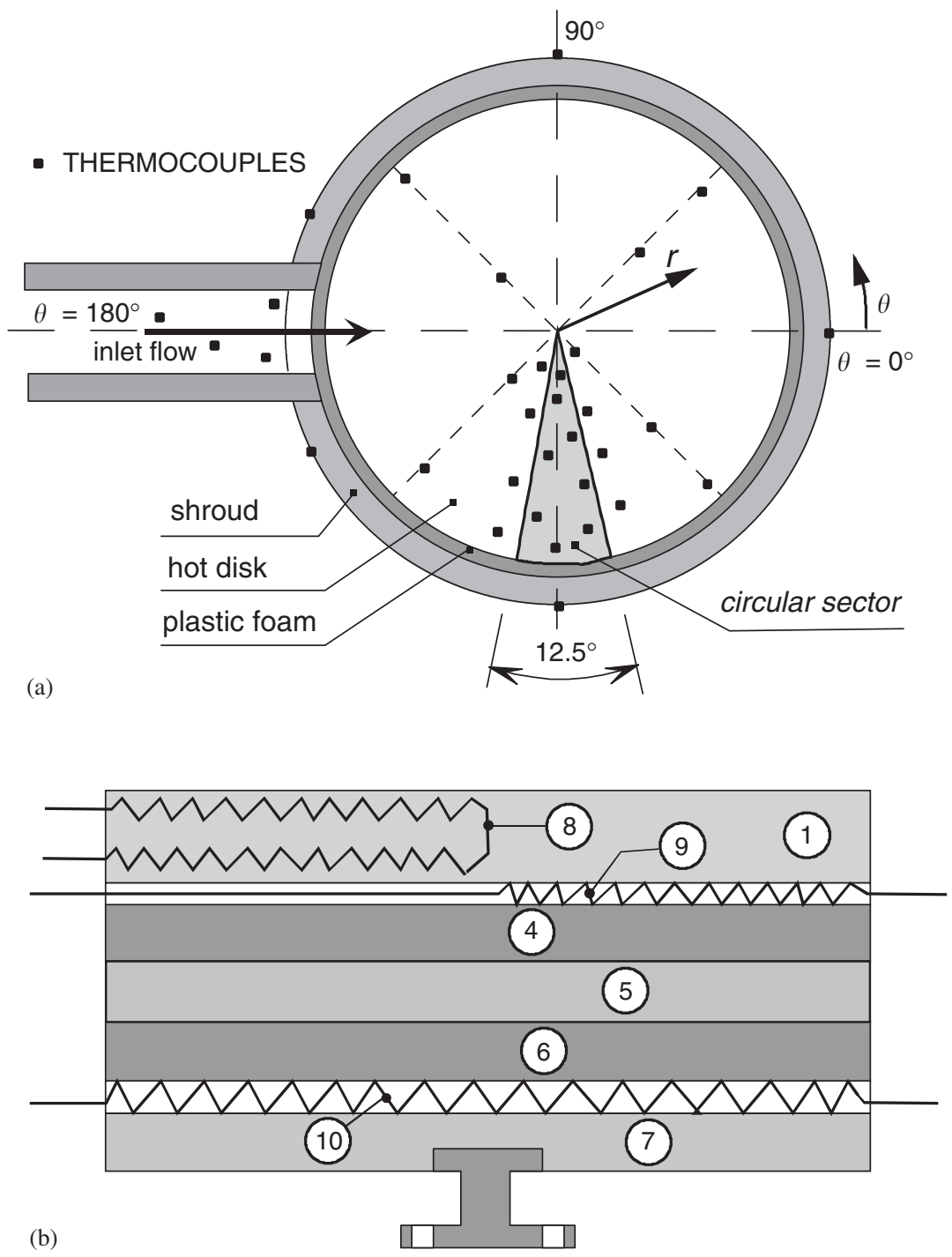

Figure 3. Diagram of hot disk at the bottom of cavity: (a) disk upper face with the circular sector and thermocouples position and (b) sandwich mounted heating system.

its original position, resulting a $12.5^{\circ}$ circular sector, as represented in Figure 3(a). Before the final assembling of the sandwich system, a plastic foam ring was inserted in the gap between its lateral surface and the fixed cylindrical Plexiglas shroud in order to prevent air leakage and minimize radial conduction heat losses, but still allowing the whole sandwich structure to rotate about its vertical axis. Thus, the angular position $\theta$ of the circular sector can be arbitrarily chosen.

By acting upon the power supply of the electric resistances (8)-(10) it is possible to reach thermal equilibrium conditions in the whole sandwich system, where disk (1) and its circular 
sector are at the same temperature and a virtual adiabatic horizontal surface results at mid height of disk (5). The power supplied to both the heating resistances (8) and (9) then allowed the calculation of the global heat transferred to the flow by convection $\left(\dot{Q}_{\mathrm{H}}\right)$ and to the other walls by radiation. Similarly, the power supplied to the circular sector was a direct measure of the heat transferred from its surface to the flow and the surroundings. Once the measured data were corrected for heat losses, the values obtained for each angular position $\theta$ of the circular sector can be used to evaluate a 'local' heat transfer flux $\left(\dot{Q}_{\mathrm{CS}}\right)$ that is representative of that $\theta$. Integration of the local values obtained for a complete revolution $(0 \leqslant \theta \leqslant 2 \pi)$ thus lead to a global value that can be compared with the global convection heat transfer mentioned above. By the application of this methodology to four independent test-cases, agreement was found within $-5.2 \%$ in the worst case and $2.8 \%$ in the best. For each test-case, reproducibility was found within $\pm 2 \%$.

The rotating disk (2) in Figure 2 is driven by an electric motor and its velocity is controlled through an electric frequency changer. The angular velocity can be chosen in the interval $40-10000 \mathrm{rpm}$. It is measured by the use of a digital tachometer.

The horizontal air jet introduced into the cavity is generated by a fan. Its mass flow rate $(\dot{m})$ is monitored by a calibrated orifice plate.

Temperatures are measured by a set of 90 thermocouples, some of which are linked in parallel, thus giving a set of 64 independent temperature sensors. The readings are performed with the aid of a data acquisition system made of a personal computer and a Metrabyte DAS-16 card with four expansion cards EXP-16.

Calibration of the devices and acquisition equipment used to measure temperature, angular velocity and inlet flow rate was previously performed.

The thermal energy flux at the inlet $\left(\dot{Q}_{\text {in }}\right)$ and outlet $\left(\dot{Q}_{\text {out }}\right)$ sections can be obtained through the measured values of the throughflow mass flux $(\dot{m})$ and mean fluid temperatures at those sections.

\section{RESULTS AND DISCUSSION}

The first aim of the tests reported in this section is to experimentally validate the theoretical method and to establish a comparative analysis on the relative performance of the three versions alternatively used to model the turbulent transport of momentum and energy within the flow. The best performing version resulting from these tests will be used in a systematic parametric study to be presented in the near future.

Dimensionless variables (hereafter denoted by a star) and parameters are introduced through the adoption of $H, \Omega R$ and $\Delta T_{\max }=T_{\max }-T_{\min }$ as references for length, velocity and temperature, respectively. As sketched in Figure $2, H$ and $R\left(=R_{1}\right)$ are the axial and radial dimensions of the flow domain and $\Omega$ is the absolute angular velocity of the rotating disk. $\Delta T_{\max }$ stands for the difference between the maximum $\left(T_{\max }=T_{\mathrm{H}}\right)$ and minimum $\left(T_{\min }=T_{\mathrm{in}}\right)$ temperature values occurring within the domain, where $T_{\mathrm{H}}$ is the bottom, hot fixed disk average temperature and $T_{\text {in }}$ is the inlet air flow temperature.

The dimensionless cavity geometrical dimensions can be defined as

$$
R^{*}=\frac{R}{H}, \quad D h_{\text {in }}^{*}=\frac{D h_{\text {in }}}{H}, \quad D h_{\text {out }}^{*}=\frac{D h_{\text {out }}}{H}
$$


where $D h_{\text {in }}$ and $D h_{\text {out }}$ are the equivalent hydraulic diameters of inlet and outlet sections, respectively.

Flow rotation and axial mass flow rate are characterized by two distinct Reynolds numbers, defined in the same order as $\operatorname{Re}_{\Omega}$ and $R e_{\mathrm{m}}$ :

$$
\begin{aligned}
& R e_{\Omega}=\frac{\rho \Omega R H}{\mu} \\
& R e_{\mathrm{m}}=\frac{\dot{m}}{\mu H}
\end{aligned}
$$

The dimensionless local velocity vector $\left(\overrightarrow{\mathbf{V}}^{*}\right)$ and the non-dimensional temperature values $\left(T^{*}\right)$ are specified as

$$
\overrightarrow{\mathbf{V}}^{*}=\frac{\overrightarrow{\mathbf{V}}}{\Omega R}
$$

and

$$
T^{*}=\frac{T-T_{\min }}{\Delta T_{\max }}
$$

respectively.

Thermal buoyancy effects are estimated through the following Grashof number $(G r)$ definition:

$$
G r=\frac{g \beta \rho^{2} H^{3} \Delta T_{\max }}{\mu^{2}}
$$

where $g=+9.81 \mathrm{~m} / \mathrm{s}^{2}, \beta$ is the volumetric thermal expansion coefficient and $\rho$ represents de fluid density.

Heat transfer between solid wall and fluid is characterized by the Nusselt number $N u$ :

$$
N u=\frac{\dot{Q} H}{A \lambda \Delta T_{\max }}
$$

where $\dot{Q}$ is the total heat transfer flux at the wall and $A$ stands for the corresponding area through which heat is transferred by convection. Global Nusselt numbers for the hot disk and its circular sector are thus noted by $N u_{\mathrm{H}}$ and $N u_{\mathrm{CS}}$, respectively.

Finally, the dimensionless thermal energy flux associated to the throughflow mass flux $\dot{m}$ at the outlet section $\left(\dot{Q}_{\text {out }}^{*}\right)$ can be specified by relating the thermal energy flux in this section $\left(\dot{Q}_{\text {out }}\right)$, the corresponding value at the inlet $\left(\dot{Q}_{\text {in }}\right)$ and the maximum heat that can be removed by the throughflow $\left(\dot{Q}_{\max }=\dot{m} c_{\mathrm{p}} \Delta T_{\max }\right)$ :

$$
\dot{Q}_{\text {out }}^{*}=\frac{\dot{Q}_{\text {out }}-\dot{Q}_{\text {in }}}{\dot{Q}_{\text {max }}}=\frac{T_{\text {out }}-T_{\text {in }}}{\Delta T_{\max }}=T_{\text {out }}^{*}
$$

where $T_{\text {out }}$ is the fluid mean temperature at the outlet section. The dimensionless thermal energy flux at the outlet section $\left(\dot{Q}_{\text {out }}^{*}\right)$ and the non-dimensional outlet fluid temperature $\left(T_{\text {out }}^{*}\right)$ are actually the same parameter. 
The fluid used for testing is air $(\operatorname{Pr}=0.704)$; the hydraulic diameter of the inlet and outlet sections, as well as the domain aspect ratio and the Grashof number are fixed and equal to $D h_{\text {in }}^{*}=0.14, D h_{\text {out }}^{*}=0.04, R^{*}=1.01$ and $G r=3.097 \times 10^{7}$, respectively. The effect of variation of these parameters will be analysed in a future work. Rotating and mass flow rate Reynolds numbers are allowed to vary with the limits: $72.6 \times 10^{3} \leqslant R e_{\Omega} \leqslant 867.7 \times 10^{3}$ and $0.0 \leqslant R e_{\mathrm{m}} \leqslant 1081.53$.

The experimental set-up described in Section 4 allows the easy monitoring of the global convection heat transfer from both the whole hot, fixed disk $\left(\dot{Q}_{\mathrm{H}}\right)$ and its circular sector $\dot{Q}_{\mathrm{CS}}$ (for different angular positions of the latter), as well as the inlet $\left(\dot{Q}_{\text {in }}\right)$ and outlet $\left(\dot{Q}_{\text {out }}\right)$ thermal energy fluxes. Uncertainty evaluation was performed for all the measurements involved with a 95\% confidence level, according to a modified Kline and McClintock method (described in Reference [15]). The relative uncertainties associated to the heat flux from the hot disk $\left(\dot{Q}_{\mathrm{H}}\right)$ and its circular sector $\left(\dot{Q}_{\mathrm{CS}}\right)$ range in the intervals $3.4-12.8 \%$ and $3.1-12.8 \%$, respectively. For the difference between the thermal energy fluxes at outlet and inlet sections $\left(\dot{Q}_{\text {out }}-\dot{Q}_{\text {in }}\right)$ the relative uncertainty is $0 \%$ when no throughflow exists $(\dot{m}=0)$ and is equal to $7.3 \%$ otherwise. Thus, the corresponding values calculated for Nusselt numbers of the whole hot disk $\left(N u_{\mathrm{H}}\right)$ and of its circular sector $\left(N u_{\mathrm{CS}}\right)$ range in the intervals 5.9$14.9 \%$ and $5.8-17.3 \%$, respectively. For the dimensionless outlet thermal energy flux $\left(\dot{Q}_{\text {out }}^{*}\right)$ the relative uncertainty is $0 \%$ when no throughflow exists and range between 0.5 and $10.1 \%$ otherwise.

Validation of the numerical procedure is thus mainly focused in the Nusselt numbers of the whole hot fixed disk $\left(N u_{\mathrm{H}}\right)$, its circular sector $\left(N u_{\mathrm{CS}}\right)$ and dimensionless outlet thermal energy flux $\left(\dot{Q}_{\text {out }}^{*}\right)$, by establishing the percentage difference $\nabla \phi=\left[\left(\phi_{\text {num }}-\phi_{\text {exp }}\right) / \phi_{\text {exp }}\right] \times 100 \%$ between numerical predictions for a general variable $\phi$ with the corresponding experimental measurements.

For the test case referred to in Section $3\left(R e_{\Omega}=144.0 \times 10^{3}, R e_{\mathrm{m}}=284.32\right)$, percentage differences between predicted and measured values of the hot disk Nusselt number and the outlet dimensionless thermal energy flux were found to be $\nabla N u_{\mathrm{H}}=-4.3 \%$ and $\nabla \dot{Q}_{\text {out }}^{*}=-9.1 \%$, respectively. These values were found acceptable for the present purpose.

Turbulent flow conditions characterized by $R e_{\Omega}=722.9 \times 10^{3}$ and $R e_{\mathrm{m}}=1081.53$ were taken as reference to analyse the relative performance of the three low Reynolds number models [Launder and Sharma (LS), Lam and Bremhorst (LB) and Lars Davidson (LD)]. The results of this analysis are summarized in Table $\mathrm{V}$, together with the effect of the modifications that were reported in Section 2: variable buoyancy term in the $\tilde{\varepsilon}$ equation $\left(f_{3}\right)$ and Yap correction (Yap). Also represented as 'Laminar' are the corresponding predictions where the turbulent transport is simply ignored.

The LS, LB and LD models lead to predictions for global flow structure and heat fluxes that are qualitatively alike. A similar behaviour is also observed in terms of numerical stability, number of iterations for convergence and influence of modifications $f_{3}$ and Yap. All of them predict wall heat fluxes and $\dot{Q}_{\text {out }}^{*}$ values that are greater than the measured ones. The $f_{3}$ correction has only a slight effect on both the convergence speed and improvement of the numerical predictions. On the other side, the Yap correction slows down convergence (almost but not exactly reached after 20000 iterations), and its effect on results depends upon which model is being used. Namely, predictions are slightly worsened with LS, somewhat enhanced with LD and substantially improved with LB. 
Table V. Validation of numerical predictions obtained with different turbulence models through comparison with experimental measurements for $\operatorname{Re}_{\Omega}=722.9 \times 10^{3}, \operatorname{Re}_{\mathrm{m}}=1081.53$ and $G r=3.097 \times 10^{7}$.

\begin{tabular}{lccccc}
\hline Model + corrections & Iterations & $N u_{\mathrm{H}}$ & $\nabla N u_{\mathrm{H}}(\%)$ & $\dot{Q}_{\text {out }}^{*}$ & $\nabla \dot{Q}_{\text {out }}^{*}(\%)$ \\
\hline Experimental & - & 146.79 & - & 0.444 & - \\
Laminar & 7960 & 102.11 & -30.4 & 0.241 & -45.8 \\
Launder and Sharma (LS) & 15417 & 172.68 & 17.6 & 0.573 & 29.0 \\
LS $+f_{3}$ & 15427 & 172.68 & 17.6 & 0.573 & 29.0 \\
LS $+f_{3}+$ Yap & 20000 & 173.88 & 18.5 & 0.579 & 30.2 \\
Lars Davidson (LD) & 13503 & 182.48 & 24.3 & 0.619 & 39.4 \\
LD $+f_{3}$ & 13359 & 182.48 & 24.3 & 0.619 & 39.3 \\
LD $+f_{3}+$ Yap & 20000 & 178.50 & 21.6 & 0.600 & 35.1 \\
Lam and Bremhorst (LB) & 10857 & 185.45 & 26.3 & 0.633 & 42.5 \\
LB $+f_{3}$ & 11065 & 185.45 & 26.3 & 0.633 & 42.5 \\
LB $+f_{3}+$ Yap & 20000 & 163.00 & 11.0 & 0.527 & 18.7 \\
\hline
\end{tabular}

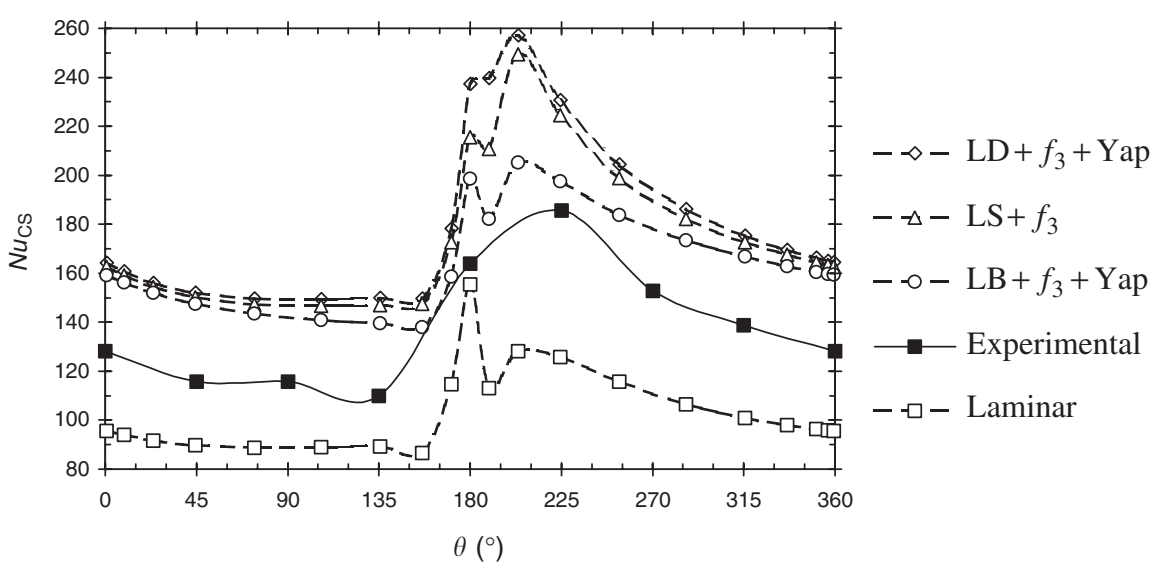

Figure 4. Comparison between experiments and predictions. Variation of the average Nusselt number of the hot disk circular sector with its angular orientation for $\operatorname{Re}_{\Omega}=722.9 \times 10^{3}$ and $\operatorname{Re}_{\mathrm{m}}=1081.53$.

Figure 4 shows a comparison between experiments and the best performing predictions obtained with each turbulence model for the variation of the average hot disk circular sector Nusselt number $\left(N u_{\mathrm{CS}}\right)$ with its angular orientation, for the situation where the inlet jet flow is located at $\theta=180^{\circ}$ and the upper disk is rotating in the positive sense. The existence and angular location of a maximum value for $N u_{\mathrm{CS}}$ is correctly detected by the numerical predictions. However its value is overestimated by all model combinations. The peak values observed for $N u_{\mathrm{CS}}$ (Figure 4) when $\theta>180^{\circ}$ are a consequence of the deflection effect due to superposition of rotation to the jet type inlet boundary. The resulting flow structure is shown in Figure 5, where two plots of the predicted flowfield are presented, one for dimensionless velocity vectors in a vertical plane (Figure 5(a)) and the other for non-dimensional 

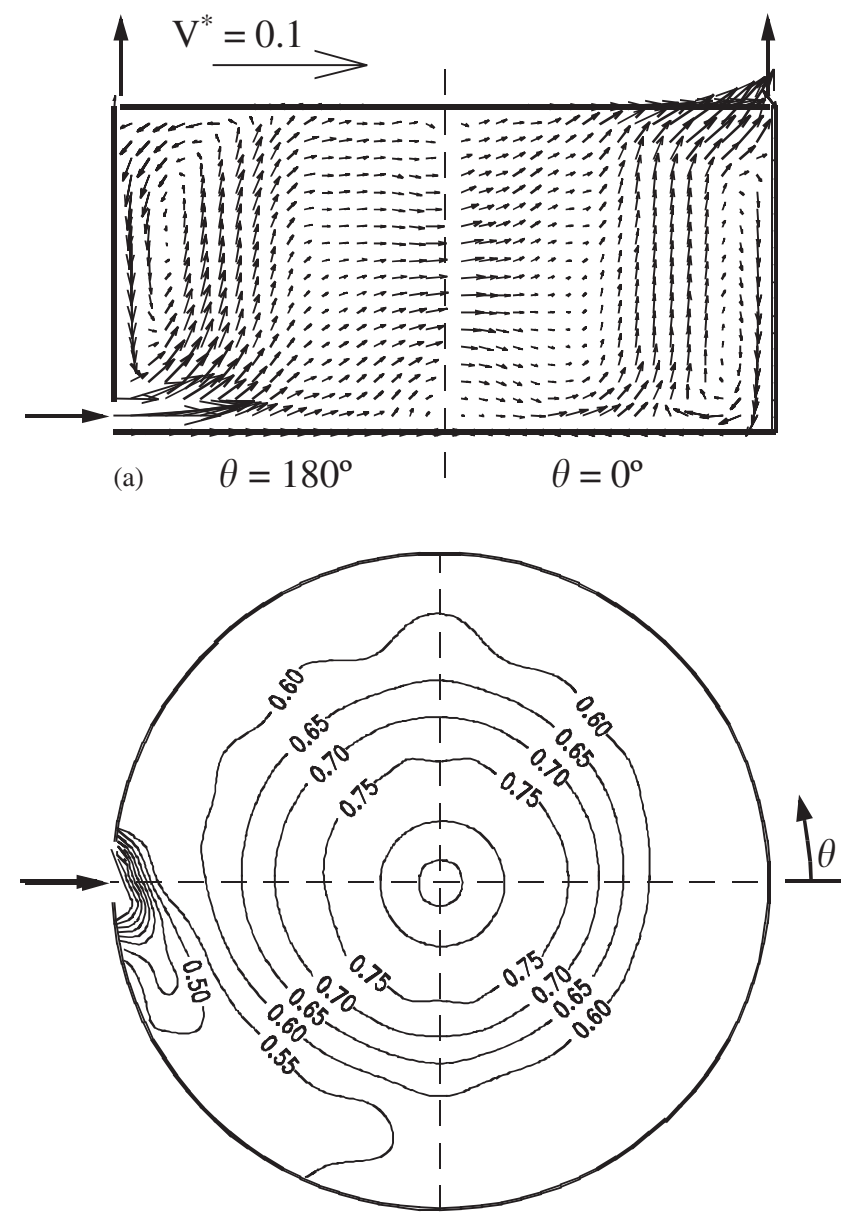

(b) $: z^{*}=0.066$

Figure 5. Computed flowfield for $R e_{\Omega}=722.9 \times 10^{3}$ and $R e_{\mathrm{m}}=1081.53$, with $\mathrm{LB}+f_{3}+$ Yap model combination: (a) dimensionless velocity vectors in a vertical plane and (b) non-dimensional temperature contours in a horizontal plane crossing the inlet jet at mid height.

temperature isolines in a horizontal plane located at $z^{*}=0.066$. Both planes contain illustrative cross-sections of the inlet airflow jet.

After a series of systematic comparisons like those in Figure 4 and in Table V, the following performance ranking was established in decreasing order: $\mathrm{LB}+f_{3}+\mathrm{Yap}, \mathrm{LS}+f_{3}$ and $\mathrm{LD}+$ $f_{3}+$ Yap. The combination LB $+f_{3}+$ Yap was thus adopted in the subsequent numerical predictions, for which additional validation is established in Table VI. Here the deviations $\nabla$ from experiments are quantified for calculations on the global Nusselt number of the hot disk $N u_{\mathrm{H}}$ and the outlet heat flux $\dot{Q}_{\text {out }}^{*}$. The comparison is made for a number of selected values of the parameters $R e_{\Omega}$ and $R e_{\mathrm{m}}$. The influence of those parameters upon $\nabla$ is seen to be important. In order to quantify the turbulence level of the flow, the numerical values obtained for the averaged dimensionless turbulent viscosity $\left(\bar{\mu}_{\mathrm{t}}^{*}\right)$ are presented in the same 
Table VI. Global comparison between experimental (Exp.) and numerical (Num.) data (LB $+f_{3}+$ Yap).

\begin{tabular}{|c|c|c|c|c|c|c|}
\hline \multicolumn{2}{|c|}{ Dimensionless parameters } & \multicolumn{2}{|c|}{ Bottom disk } & \multicolumn{2}{|c|}{ Outlet flow } & \multirow[b]{2}{*}{$\begin{array}{l}\text { Mean turbulen } \\
\text { viscosity }\end{array}$} \\
\hline Rotation & Mass flow rate & Exp. & Num. (\%) & Exp. & Num. (\%) & \\
\hline$R e_{\Omega}$ & $R e_{\mathrm{m}}$ & $N u_{\mathrm{H}}$ & $\nabla N u_{\mathrm{H}}$ & $\dot{Q}_{\text {out }}^{*}$ & $\nabla \dot{Q}_{\text {out }}^{*}$ & $\bar{\mu}_{t}^{*}$ \\
\hline 0.0 & 0.0 & 16.72 & -6.9 & & 0.0 & 0.0 \\
\hline $72.6 \times 10^{3}$ & 0.0 & 36.63 & 13.1 & & 0.0 & 0.0 \\
\hline $144.0 \times 10^{3}$ & 0.0 & 44.42 & 24.1 & & 0.0 & 0.0 \\
\hline $216.7 \times 10^{3}$ & 0.0 & 56.51 & 22.5 & & 0.0 & 0.003 \\
\hline $289.0 \times 10^{3}$ & 0.0 & 67.37 & 19.0 & & 0.0 & 0.32 \\
\hline $723.1 \times 10^{3}$ & 0.0 & 105.72 & 23.7 & & 0.0 & 0.99 \\
\hline $867.7 \times 10^{3}$ & 0.0 & 108.10 & 33.2 & & 0.0 & 1.18 \\
\hline 0.0 & 284.32 & 34.95 & -14.2 & 0.242 & -23.7 & 0.0 \\
\hline $72.6 \times 10^{3}$ & 284.32 & 38.67 & -9.2 & 0.243 & -14.8 & 0.0 \\
\hline $144.0 \times 10^{3}$ & 284.32 & 51.71 & -4.3 & 0.285 & -9.1 & 0.0 \\
\hline $216.7 \times 10^{3}$ & 284.32 & 62.49 & -5.3 & 0.312 & -7.3 & 0.001 \\
\hline $289.1 \times 10^{3}$ & 284.32 & 71.16 & -1.2 & 0.337 & 6.1 & 6.26 \\
\hline $723.1 \times 10^{3}$ & 284.32 & 122.32 & 4.1 & 0.500 & 14.0 & 1484.9 \\
\hline $867.1 \times 10^{3}$ & 284.32 & 123.73 & 6.4 & 0.550 & 18.8 & 4383.0 \\
\hline 0.0 & 721.95 & 60.23 & -29.0 & 0.242 & -40.2 & 0.04 \\
\hline $72.6 \times 10^{3}$ & 721.95 & 56.01 & -22.1 & 0.238 & -34.0 & 0.09 \\
\hline $144.0 \times 10^{3}$ & 721.95 & 49.43 & -21.0 & 0.230 & -28.6 & 0.11 \\
\hline $216.4 \times 10^{3}$ & 721.95 & 62.17 & -14.2 & 0.250 & -19.8 & 1.35 \\
\hline $289.1 \times 10^{3}$ & 721.95 & 71.59 & 25.6 & 0.273 & 27.5 & 6.26 \\
\hline $722.9 \times 10^{3}$ & 721.95 & 136.56 & 13.0 & 0.487 & 29.2 & 1491.7 \\
\hline $866.6 \times 10^{3}$ & 721.95 & 146.26 & 11.3 & 0.536 & 31.7 & 4496.7 \\
\hline 0.0 & 1081.53 & 68.05 & -37.5 & 0.254 & -51.7 & 0.35 \\
\hline $72.6 \times 10^{3}$ & 1081.53 & 75.02 & -29.9 & 0.246 & -43.8 & 0.51 \\
\hline $143.7 \times 10^{3}$ & 1081.53 & 72.65 & -27.6 & 0.237 & -40.2 & 2.27 \\
\hline $216.4 \times 10^{3}$ & 1081.53 & 60.63 & 46.0 & 0.218 & 66.4 & 3.39 \\
\hline $289.4 \times 10^{3}$ & 1081.53 & 67.15 & 34.3 & 0.234 & 52.2 & 6.13 \\
\hline $722.9 \times 10^{3}$ & 1081.53 & 146.79 & 11.0 & 0.444 & 18.7 & 1216.2 \\
\hline $866.6 \times 10^{3}$ & 1081.53 & 160.01 & 22.3 & 0.505 & 43.1 & 4563.8 \\
\hline
\end{tabular}

table, according to the following definitions:

$$
\begin{aligned}
& \mu_{\mathrm{t}}^{*}=\frac{\mu_{\mathrm{t}}}{\mu} \\
& \bar{\mu}_{\mathrm{t}}^{*}=\left(\sum_{i=1}^{n} \mu_{\mathrm{t}}^{*} V o l_{i}\right) / V o l
\end{aligned}
$$

where $n$ is the number of real nodes in the calculation domain, $V_{o l}$ represents the volume associated with each node and $\mathrm{Vol}$ stands for the inside volume of the entire cavity. 
As shown in Table VI, in the absence of throughflow, $\nabla N u_{\mathrm{H}}$ values are positive and tend to increase with increasing $R e_{\Omega}$. A different behaviour is observed when rotation and axial flow are both present. The extreme positive and negative $\nabla$ values pointed out in Table VI are above the experimental uncertainty. Deviations of the present numerical predictions may be attributed to different factors, namely: the use of turbulence models improved with corrections that were not specifically calibrated for the present conditions; the well-known difficulty in reproducing in laboratory the exact boundary conditions (namely temperature distribution) used for numerical calculations; the experimental uncertainty itself. Even though improvement is thus still necessary, agreement between both approaches is seen to be very satisfactory in most of the flow conditions analysed. This means that further interesting predictions may be obtained with confidence by using the present numerical tool.

In fact, a systematic parametrical analysis of the flow configuration and heat transfer phenomena, using the selected $\mathrm{LB}+f_{3}+$ Yap combination to model the turbulent transport is now being carried out and will be reported in a future work.

\section{CONCLUSIONS}

A numerical procedure of the control-volume type aimed at modelling steady three-dimensional rotating flows with heat transfer, in both laminar and turbulent conditions was developed and successfully tested by comparison with measurements obtained in a laboratory rig. The theoretical approach formerly included three alternative versions of low Reynolds number, $k-\varepsilon$ type models that were then adjusted to include the effects of rotation, thermal buoyancy and streamline curvature, as well as the turbulence length-scale limitation known as 'Yap correction'. After validation, they were classified in terms of relative performance. The best performing combination of this ranking (noted 'LB $+f_{3}+$ Yap') was then selected and thoroughly tested for different flow conditions that were characterized in terms of the rotating and mass flow rate Reynolds numbers. With the exception of a number of particular situations, very satisfactory agreement was observed between numerical predictions and the corresponding measurements, thus providing the basis for a further systematic study on the dependence of flow structure and heat transport upon the most relevant dynamic and thermal flow parameters.

\section{APPENDIX A: NOMENCLATURE}

$\begin{array}{ll}A & \text { Area }\left(\mathrm{m}^{2}\right) \\ B_{U}, B_{V}, B_{W} & \text { buoyancy source terms in } U, V, W \text { equations (Equation (3)) } \\ B_{k}, E_{\varepsilon} & \text { extra-terms in the } k \text { and } \varepsilon \text { equations (Table IV) } \\ C_{1}, C_{2}, C_{3}, C_{\mu}, \sigma_{k}, \sigma_{\varepsilon} & \text { constants of turbulence model (Table II) } \\ c_{\mathrm{p}} & \text { heat capacity at constant pressure }(\mathrm{J} /(\mathrm{kg} \mathrm{K})) \\ D h_{\mathrm{in}}, D h_{\text {out }} & \text { equivalent hydraulic diameters of inlet and outlet sections (m) } \\ D_{k}, D_{\varepsilon} & \text { dissipation sources of } k \text { and } \varepsilon \text { equations (Equations (7), (9)) } \\ f_{\mu}, f_{1}, f_{2} & \text { damping functions of turbulence model (Table III) } \\ f_{3}, f_{\mathrm{c}}, f_{\mathrm{r}} & \text { empirical turbulence functions (Equations (8), (9), (10)) } \\ G_{k}, G_{\varepsilon} & \text { buoyancy sources of } k \text { and } \varepsilon \text { equations (Equations (7), (8)) } \\ G r & \text { Grashof number (Equation (19)) }\end{array}$




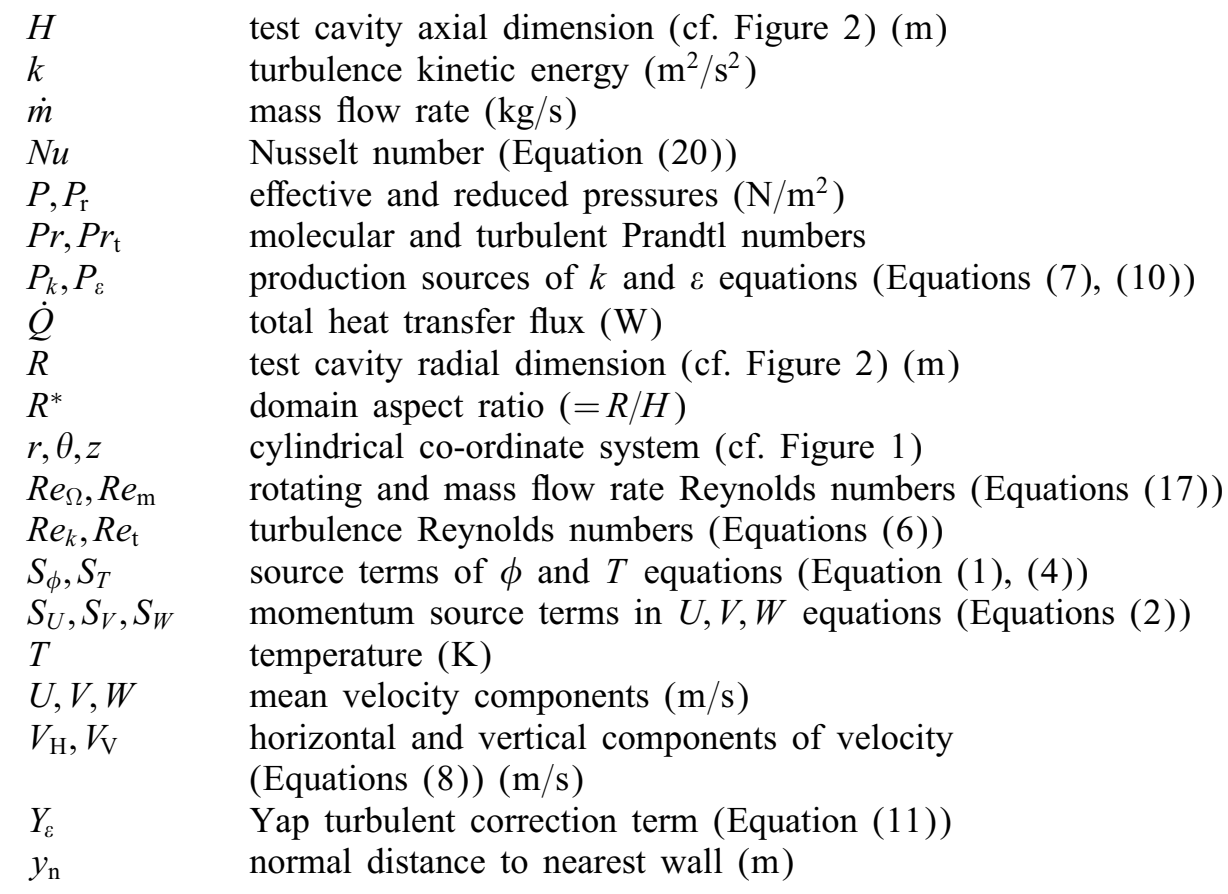

\section{Greek letters}

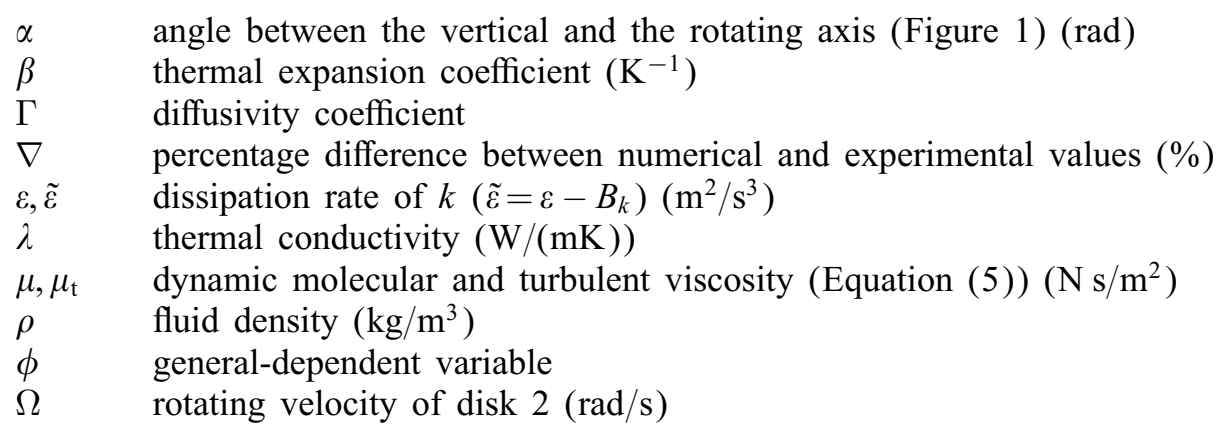

\section{Superscripts}

$k, P, T, U, V, W, \varepsilon, \phi \quad$ relative to $k, P, T, U, V, W, \varepsilon$ and $\phi$ equations $*,+\quad$ dimensionless variable

\section{Subscripts}

$\begin{array}{ll}\text { ef } & \text { effective } \\ \text { CS } & \text { circular sector of hot disk } \\ \text { H } & \text { hot disk (disk 1 of Figure 2) } \\ \text { in, out } & \text { inlet and outlet conditions } \\ \text { min, max } & \text { minimum and maximum values }\end{array}$




\section{REFERENCES}

1. Owen JM, Rogers RH. Flow and Heat Transfer in Rotating-Disc Systems, vol. 1: Rotor-Stator Systems \& vol. 2: Rotating Cavities. Wiley, Inc.: Chichester, England, vol. 1: 1989 and vol. 2: 1995.

2. Lee $\mathrm{CH}$, Hyun JM. Flow of a stratified fluid in a cylinder with a rotating lid. International Journal of Heat and Fluid Flow 1999; 20:26-33.

3. Long CA, Morse A, Tucker P. Measurement and computation of heat transfer in high-pressure compressor drum geometries with axial throughflow. ASME Journal of Turbomachinery 1997; 119:51-60.

4. Herrero J, Girald F, Humphrey JAC. Non-isothermal laminar flow and heat transfer between disks corotating in a fixed enclosure. International Journal of Heat and Mass Transfer 1999; 42:3291-3306.

5. El-Oun ZD, Neller PH, Turner AB. Sealing of a shrouded rotor-stator system with pre-swirl coolant. $A S M E$ Journal of Turbomachinery 1988; 110:218-228.

6. Chew JW. A theoretical study of ingress for shrouded rotating disc systems with radial outflow. ASME Journal of Turbomachinery 1991; 113:91-97.

7. Chen HC, Patel VC. Near-wall turbulence models for complex flows including separation. AIAA Journal 1988; 26(6):641-648

8. Nallasamy M. Turbulence models and their applications to the prediction of internal flows: a review. Computers and Fluids 1987; 15(2):151-194.

9. Launder BE, Sharma BI. Application of the energy-dissipation model of turbulence to the calculation of flow near a spinning disc. Letters in Heat and Mass Transfer 1974; 1:131-138.

10. Lam CKG, Bremhorst K. A modified form of the $k-\varepsilon$ model for predicting wall turbulence. Journal of Fluids Engineering 1981; 103:456-460.

11. Davidson L. Calculation of the turbulent buoyancy-driven flow in a rectangular cavity using an efficient solver and two different low Reynolds number $k-\varepsilon$ turbulent models. Numerical Heat Transfer Part A 1990; 18: $129-147$.

12. Gray DD, Giorgini A. The validity of the Boussinesq approximation for liquids and gases. International Journal of Heat and Mass Transfer 1976; 19:545-551.

13. Zografos AI, Martin WA, Sunderland JE. Equations of properties as a function of temperature for seven fluids. Computer Methods in Applied Mechanics and Engineering 1987; 61:177-187.

14. Oliveira LA. Contribution to the study of axisymmetric flow between rotating disks. Ph.D. Thesis, Faculty of Sciences and Technology, University of Coimbra, Portugal, 1986 (in portuguese).

15. Raimundo AM. Contribution to the study of rotating flows: heat transfer analysis in three-dimensional nonaxisymmetric conditions. Ph.D. Thesis, Faculty of Sciences and Technology, University of Coimbra, Portugal, 1998 , (in portuguese).

16. Costa JJ, Oliveira LA, Blay D. Test of several versions for the $k-\varepsilon$ type turbulence modelling of internal mixed convection flows. International Journal of Heat and Mass Transfer 1999; 42:4391-4409.

17. Launder BE, Priddin CH, Sharma BI. The calculation of turbulent boundary layers on spinning and curved surfaces. Journal of Fluids Engineering 1977; 99:231-239.

18. Yap C. Turbulent heat and momentum transfer in recirculating and impinging flows. Ph.D. Thesis, Faculty of Technology, University of Manchester, United Kingdom, 1987.

19. Patankar SV. Numerical Heat Transfer and Fluid Flow. Hemisphere Publishing Corporation: Washington DC, USA, 1980.

20. Van Doormaal J, Raithby GD. Enhancements of the SIMPLE method for predicting incompressible fluid flows. Numerical Heat Transfer 1984; 7:147-163. 